(C) 2011 IEEE. Personal use of this material is permitted. Permission from IEEE must be obtained for all other uses, in any current or future media, including reprinting/republishing this material for advertising or promotional purposes, creating new collective works, for resale or redistribution to servers or lists, or reuse of any copyrighted component of this work in other works. 


\section{Bit Error Rate Performance of MIMO MMSE Receivers in Correlated Rayleigh Flat-Fading Channels}

Li Hong and Ana Garcia Armada, Senior Member, IEEE

\begin{abstract}
This paper analyzes the average bit error rate (BER) of multiple-input-multiple-output (MIMO) systems in transmit-correlated Rayleigh flat-fading channels. The receiver scheme is based on the minimum mean square error (MMSE) criterion, and the input may be precoded to optimize the communication. Accurate closed-form formulations for the average BER are derived.
\end{abstract}

Index Terms-Bit error rate (BER), correlated channel, Gamma distribution, minimum mean square error (MMSE), multipleinput-multiple-output (MIMO), signal-to-interference-plus-noise ratio (SINR).

\section{INTRODUCTION}

Multiple-input-multiple-output (MIMO) techniques are adopted in current wireless standards to improve their performance in terms of capacity and bit error rate (BER). In this paper, we focus on linear receiver schemes. Among these schemes, the minimum mean square error (MMSE) receiver is a good choice, because it offers better performance than zero forcing $(\mathrm{ZF})$, avoiding the noise-enhancement effect of ZF. In MIMO systems, the adaptation of the modulation at the transmitter side according to the channel characteristics allows for reducing the transmission power and/or enhancing the data rates [1]. To do so, a closed-form expression is needed, relating the average BER and the required transmission power for different candidate modulation schemes. Moreover, the transmitted signal may be optimized under other different criteria using a precoder [2]. A closed-form expression for the average BER is also useful, in general, for the system design, even if adaptive modulation or precoding is not used.

In this paper, we analyze the performance of a general MIMO system, with or without precoding, in transmit-correlated Rayleigh flatfading channels with an MMSE receiver. Our analysis will be based on the signal-to-interference-plus-noise ratio (SINR) distribution. It is proved in [3] that the SINR after a ZF receiver is a Gamma random variable when a uniform power distribution is used in the transmitter. Making use of this distribution in [1], we obtained the average BER when a $\mathrm{ZF}$ scheme is employed at the receiver. With regard to the MMSE receiver, some expressions have been developed, which are only accurate for high dimensions [4] or are particularized to diversitycombining receivers [5]. In [6], the performance is evaluated for large dimensions in terms of mutual information. None of these works contemplates the precoder.

This work was supported in part by Project TEC2008-06327-C03-02, Project CSD200800010, and Project CCG08-UC3M/TIC-4069.

The authors are with the Department of Signal Theory and Communications, Universidad Carlos III de Madrid, 28911 Leganés, Spain (e-mail: lihong@tsc.uc3m.es; agarcia@tsc.uc3m.es).
Recently, the distribution of the SINR after an MMSE receiver has been obtained for the case of a transmit-correlated Rayleigh flat-fading channel [7]. The authors show that the SINR approaches a Gamma or generalized Gamma distribution. In this paper, we will make use of both distributions to obtain the average BER for a MIMO system that employs $M$-ary quadrature-amplitude modulation ( $M$-QAM) and show their accuracy, even for a small number of antennas. We will compare them to the expressions obtained in [4], which are accurate for large antenna dimensions, and [8], where the authors also use a generalized Gamma distribution. However, its parameters are obtained following a different procedure and assumptions [9], and the developed expressions are accurate for both large and small dimensions but, in this last case, only for small signal-to-noise ratios (SNRs), as shown in [8]. In addition, in [8], the BER is averaged over all the MIMO transmitted streams, whereas in channels that exhibit high correlation, the use of a precoder may lead to substantial differences among the spatially multiplexed streams. Therefore, we present several approximations that may be chosen depending on the accuracy and complexity requirements of the adaptation algorithms used to reduce the transmission power and/or enhance the data rates.

Notation: $\mathbf{I}_{\mathbf{N}}$ denotes the $N \times N$ identity matrix, $\operatorname{diag}(\mathbf{x})$ denotes a diagonal matrix with the vector $\mathbf{x}$ elements in its diagonal and 0 elsewhere, and $\mathbf{A}^{\dagger}$ is the conjugate transpose of matrix $\mathbf{A}$.

\section{System ModeL}

Let $n_{\mathrm{T}}$ and $n_{\mathrm{R}}$ denote, respectively, the number of transmit and receive antennas. The input is the $n_{\mathrm{T}} \times 1$ complex vector

$$
\mathbf{x}=\sqrt{\mathbf{P}} \mathbf{V s}
$$

where $\mathbf{s}$ is a $n_{\mathrm{T}} \times 1$ vector of unit-energy $M$-QAM data. The unitary $n_{\mathrm{T}} \times n_{\mathrm{T}}$ matrix $\mathbf{V}$ is a linear precoder whose columns define the signaling vectors, whereas the diagonal $n_{\mathrm{T}} \times n_{\mathrm{T}}$ matrix $\mathbf{P}$ determines the fraction of the available power allocated to each such vector. The $n_{\mathrm{R}} \times 1$ received vector is

$$
\mathbf{y}=\mathbf{H}_{\mathbf{w}} \sqrt{\mathbf{R}_{\mathbf{t}}} \mathbf{x}+\mathbf{n}
$$

where the channel matrix has correlation at the transmit side, expressed by the transmit correlation matrix $\mathbf{R}_{\mathbf{t}}$, and $\mathbf{H}_{\mathbf{w}}$ of dimensions $n_{\mathrm{R}} \times n_{\mathrm{T}}$ has independent and identically distributed (i.i.d.) circularly symmetric complex Gaussian entries with zero mean and unit variance. Finally, $\mathbf{n}$ is a zero-mean circularly symmetric complex Gaussian $n_{\mathrm{R}} \times 1$ noise vector.

We define an effective channel matrix as follows:

$$
\mathbf{H}=\mathbf{H}_{\mathrm{w}} \sqrt{\mathbf{R}_{\mathrm{t}}} \sqrt{\mathbf{P}}=\mathbf{H}_{\mathrm{c}} \sqrt{\mathbf{P}} .
$$

Similar to [7], the power matrix is defined as

$$
\mathbf{P}=\operatorname{diag}\left(\left[p_{1}, \ldots, p_{n_{\mathrm{T}}}\right]\right), p_{i}=\pi_{i} \gamma \frac{n_{\mathrm{R}}}{n_{\mathrm{T}}}, \quad i=1, \ldots, n_{\mathrm{T}}
$$

where $\gamma$ is the average $\mathrm{SNR}$, i.e.,

$$
\gamma=\frac{E\left\{\mathbf{x}^{\dagger} \mathbf{x}\right\}}{E\left\{\mathbf{n}^{\dagger} \mathbf{n}\right\}}
$$

and $\pi_{i}$ specifies the fraction of the available power allocated to each signaling vector (also denoted here as a transmitted stream). 
The MMSE receiver yields at its output $\mathbf{z}=\mathbf{G y}$ with

$$
\mathbf{G}=\left(n_{\mathrm{T}} \mathbf{I}_{\mathbf{n}_{\mathrm{T}}}+(\mathbf{H V})^{\dagger}(\mathbf{H V})\right)^{-1}(\mathbf{H V})^{\dagger}
$$

Then, the SINR for the $k$ th stream after the MMSE receiver is [10]

$$
\operatorname{SINR}_{k}=\frac{1}{\left[\left(\mathbf{I}_{\mathbf{n}_{\mathrm{T}}}+\frac{1}{n_{\mathrm{R}}}(\mathbf{H V})^{\dagger}(\mathbf{H V})\right)^{-1}\right]_{k, k}}-1
$$

In case precoding is not used, then $\mathbf{V}=\mathbf{I}_{\mathbf{n}_{\mathrm{T}}}$, and $\pi_{i}=1$, $i=1, \ldots, n_{\mathrm{T}}$.

\section{Average Bit Error Rate Assuming A GAMMA DISTRIBUTION OF THE SignAL-TO-INTERFERENCE-PLUS-NOISE RATIO}

According to [7], the $\mathrm{SINR}_{k}$ of each stream (7) can be approximated by a Gamma distribution as

$$
f_{\operatorname{SINR}_{k}}(s)=\frac{s^{\alpha_{k}-1} e^{-s / \theta_{k}}}{\Gamma\left(\alpha_{k}\right) \theta_{k}^{\alpha_{k}}} .
$$

The parameters of the Gamma distribution (8) can be written as (the subindex $k$ is dropped hereafter for notational simplicity)

$$
\begin{gathered}
\alpha=\frac{\left(n_{\mathrm{R}}-n_{\mathrm{T}}+1+\left(n_{\mathrm{T}}-1\right) \mu\right)^{2}}{n_{\mathrm{R}}-n_{\mathrm{T}}+1+\left(n_{\mathrm{T}}-1\right) \sigma^{2}} \\
\theta=\frac{\boldsymbol{\Sigma}}{n_{\mathrm{R}}} \frac{n_{\mathrm{R}}-n_{\mathrm{T}}+1+\left(n_{\mathrm{T}}-1\right) \sigma^{2}}{n_{\mathrm{R}}-n_{\mathrm{T}}+1+\left(n_{\mathrm{T}}-1\right) \mu}
\end{gathered}
$$

where $\boldsymbol{\Sigma}$ for the $k$ th stream is

$$
\boldsymbol{\Sigma}=\frac{1}{\left[\mathbf{R}^{-1}\right]_{k, k}}
$$

with the generalized covariance matrix defined as $\mathbf{R}=$ $\sqrt{\mathbf{P}} \mathbf{V R}_{\mathbf{t}} \mathbf{V}^{\dagger} \sqrt{\mathbf{P}}$. In addition, $\mu$ and $\sigma^{2}$ must be obtained by solving the following equations [7]:

$$
\begin{aligned}
\mu & =\frac{1}{n_{\mathrm{T}}-1} \sum_{i=1}^{n_{\mathrm{T}}-1} \frac{1}{\tau_{i}(1-v+v \mu)+1} \\
\sigma^{2} & \left(1+\frac{1}{n_{\mathrm{T}}-1} \sum_{i=1}^{n_{\mathrm{T}}-1} \frac{\tau_{i} v}{\left(\tau_{i}(1-v+v \mu)+1\right)^{2}}\right) \\
& =\frac{1}{n_{\mathrm{T}}-1} \sum_{i=1}^{n_{\mathrm{T}}-1} \frac{\tau_{i} v \mu+1}{\left(\tau_{i}(1-v+v \mu)+1\right)^{2}}
\end{aligned}
$$

where $\tau_{i}$ are the eigenvalues of $\mathbf{R}_{(-k,-k)}$, and $v=\left(n_{\mathrm{T}}-1\right) / n_{\mathrm{R}}$. $\mathbf{R}_{(-k,-k)}$ is the generalized covariance matrix $\mathbf{R}$, with the $k$ th row and the $k$ th column removed.

Then, the average BER for the $k$ th signal can be obtained as

$$
\bar{P}_{e}(\alpha, \theta)=\int_{0}^{\infty} P_{e}(s) \frac{s^{\alpha-1} e^{-s / \theta}}{\Gamma(\alpha) \theta^{\alpha}} d s
$$

where $\Gamma(\cdot)$ is the complete Gamma function, and $P_{e}(\cdot)$ is the instantaneous BER function for the corresponding scalar signal, which depends on the modulation scheme used at the transmitter.
Accurate expressions for $\bar{P}_{e}(\alpha, \theta)$ in (13) have been obtained in [1] for $M$-QAM as follows.

For 2-pulse amplitude modulation (2-PAM), we have

$$
\begin{aligned}
\bar{P}_{e}=\frac{1}{2 \Gamma(\alpha)}\left[\Gamma(\alpha)-2 \sqrt{\frac{\theta}{\pi}} \Gamma\left(\frac{1}{2}+\alpha\right)\right. \\
\\
\left.\quad \times{ }_{2} F_{1}\left(\frac{1}{2}, \frac{1}{2}+\alpha ; \frac{3}{2} ;-\theta\right)\right]
\end{aligned}
$$

whereas for 4-QAM, we have

$$
\begin{aligned}
\bar{P}_{e}=\frac{1}{2 \Gamma(\alpha)}\left[\Gamma(\alpha)-\sqrt{\frac{2 \theta}{\pi}} \Gamma\right. & \left(\frac{1}{2}+\alpha\right) \\
& \left.\times{ }_{2} F_{1}\left(\frac{1}{2}, \frac{1}{2}+\alpha ; \frac{3}{2} ;-\frac{\theta}{2}\right)\right]
\end{aligned}
$$

and for $M$-QAM, we have

$$
\bar{P}_{e}=\frac{\Gamma(\alpha)-\sqrt{\frac{6 \theta}{\pi(M-1)}} \Gamma\left(\frac{1}{2}+\alpha\right){ }_{2} F_{1}\left(\frac{1}{2}, \frac{1}{2}+\alpha ; \frac{3}{2} ; \frac{-3 \theta}{2(M-1)}\right)}{1 / 2 \log _{2}(M) \Gamma(\alpha)}
$$

where ${ }_{2} F_{1}$ is the hypergeometric function, i.e.,

$$
{ }_{2} F_{1}(a, b ; c ; d)=\frac{\Gamma(c)}{\Gamma(b) \Gamma(c-b)} \int_{0}^{1} \frac{t^{b-1}(1-t)^{c-b-1}}{(1-t d)^{a}} d t .
$$

Note that, although $M$-QAM includes 2-PAM and 4-QAM, the expression in (16) is an approximation that is valid for high $M$, whereas (14) and (15) are accurate. These expressions are simplified for the high-SNR regime as follows [1].

For 2-PAM, we have

$$
\bar{P}_{e} \approx \frac{1}{2 \sqrt{\pi}}(1+\theta)^{-\alpha}
$$

whereas for 4-QAM, we have

$$
\bar{P}_{e} \approx \frac{1}{2 \sqrt{\pi}}\left(1+\frac{\theta}{2}\right)^{-\alpha}
$$

and for $M$-QAM, we have

$$
\bar{P}_{e} \approx \frac{1}{\log _{2} M} \frac{2}{\sqrt{\pi}}\left(1+\frac{3 \theta}{2(M-1)}\right)^{-\alpha} .
$$

\section{Average Bit Error Rate by Generalized Gamma Distribution for Minimum Mean Square ERror}

It is shown in [7] that the SINR of each stream can more accurately be approximated by a generalized Gamma distribution as

$$
f_{\mathrm{SINR}}(s)=\frac{\xi s^{\alpha \xi-1} e^{-\left(\frac{s}{\theta}\right)^{\xi}}}{\Gamma(\alpha) \theta^{\alpha \xi}}
$$

with the same parameters $\alpha$ and $\theta$ and a third parameter

$$
\xi=\frac{2\left(1-\frac{n_{\mathrm{T}}-1}{n_{\mathrm{R}}}+\frac{n_{\mathrm{T}}-1}{n_{\mathrm{R}}} \mu\right)\left(1-\frac{n_{\mathrm{T}}-1}{n_{\mathrm{R}}}+\frac{n_{\mathrm{T}}-1}{n_{\mathrm{R}}} \eta\right)}{\left(1-\frac{n_{\mathrm{T}}-1}{n_{\mathrm{R}}}+\frac{n_{\mathrm{T}}-1}{n_{\mathrm{R}}} \sigma^{2}\right)^{2}}-1
$$


TABLE I

Average BER From the Generalized Gamma Distribution

\begin{tabular}{|c|c|}
\hline & If $\xi>1$ \\
\hline 2-PAM & $\bar{P}_{e}=\frac{1}{2 \sqrt{\pi}} \exp \left(\frac{\alpha}{\xi-1}\left(1-(1+\theta \xi)^{\frac{\xi-1}{\xi}}\right)\right)$ \\
\hline 4-QAM & $\bar{P}_{e}=\frac{1}{2 \sqrt{\pi}} \exp \left(\frac{\alpha}{\xi-1}\left(1-\left(1+\frac{\theta \xi}{2}\right)^{\frac{\xi-1}{\xi}}\right)\right)$ \\
\hline$M$-QAM & $\bar{P}_{e}=\frac{2}{\log _{2}(M) \sqrt{\pi}} \exp \left(\frac{\alpha\left(1-\left(1+\frac{3}{2(M-1)} \theta \xi\right)^{\frac{\xi-1}{\xi}}\right)}{\xi-1}\right)$ \\
\hline 2-PAM & $\bar{P}_{e}=\frac{1}{2 \sqrt{\pi}} \exp \left(\frac{\alpha}{1-\xi}\left(\left(\frac{1}{1+\theta \xi}\right)^{\frac{1-\xi}{\xi}}-1\right)\right)$ \\
\hline 4-QAM & $\bar{P}_{e}=\frac{1}{2 \sqrt{\pi}} \exp \left(\frac{\alpha}{1-\xi}\left(\left(\frac{1}{1+\frac{\theta \xi}{2}}\right)^{\frac{1-\xi}{\xi}}-1\right)\right)$ \\
\hline$M$-QAM & $\bar{P}_{e}=\frac{2}{\log _{2}(M) \sqrt{\pi}} \exp \left(\frac{\alpha\left(\frac{1}{1+\frac{3}{2(M-1)}}\right)^{\frac{1-\xi}{\xi}}-1}{1-\xi}\right)$ \\
\hline
\end{tabular}

TABLE II

LOW-SNR AVERAGE BER From THE GENERALIZED GAMMA DISTRIBUTION

\begin{tabular}{|c|c|}
\hline 2-PAM & $\bar{P}_{e} \approx \frac{1}{2 \sqrt{\pi}}\left(1+\left(\frac{1}{\theta}\right)^{\xi}\right)^{-\alpha}\left(\frac{1}{\theta}\right)^{\alpha \xi}$ \\
\hline 4-QAM & $\bar{P}_{e} \approx \frac{1}{2 \sqrt{\pi}}\left(\frac{1}{2}+\left(\frac{1}{\theta}\right)^{\xi}\right)^{-\alpha}\left(\frac{1}{\theta}\right)^{\alpha \xi}$ \\
\hline$M$-QAM & $\bar{P}_{e} \approx \frac{2}{\log _{2}(M) \sqrt{\pi}}\left(\frac{3}{2(M-1)}+\left(\frac{1}{\theta}\right)^{\xi}\right)^{-\alpha}\left(\frac{1}{\theta}\right)^{\alpha \xi}$ \\
\hline
\end{tabular}

with the same $\mu$ and $\sigma^{2}$ obtained with (11) and (12) and $\eta$ obtained by solving [7]

$$
\begin{gathered}
\eta\left(1+\frac{1}{n_{\mathrm{T}}-1} \sum_{i=1}^{n_{\mathrm{T}}-1} \frac{\tau_{i} v}{\left(\tau_{i}(1-v+v \mu)+1\right)^{2}}\right) \\
=\frac{2}{n_{\mathrm{T}}-1}\left(\sum _ { i = 1 } ^ { n _ { \mathrm { T } } - 1 } \left(\frac{\tau_{i} v \sigma^{2}}{\left(\tau_{i}(1-v+v \mu)+1\right)^{2}}\right.\right. \\
\left.\left.+\frac{\left(\tau_{i} v \mu-\tau_{i} v \sigma^{2}+1\right)^{2}}{\left(\tau_{i}(1-v+v \mu)+1\right)^{3}}\right)\right)
\end{gathered}
$$

The average BER can be calculated from the generalized Gamma distribution using its moment-generating function (MGF), as shown in Appendix A, giving the expressions in Table I. For the low-SNR regime, these expressions can further be simplified (see Appendix B) and are given in Table II.

Again, we would like to emphasize that, although $M$-QAM includes 2-PAM and 4-QAM, the expressions shown for $M$-QAM in Tables I and II are approximations that are valid for high $M$, whereas the expressions shown for 2-PAM and 4-QAM are more accurate for these particular cases.

\section{NUMERICAL RESULTS}

To illustrate the accuracy of the developed expressions, we compare them with Monte Carlo simulation results in a $4 \times 4$ MIMO system with an MMSE receiver, where we assume that the transmit correlation matrix has elements $R_{t_{i j}}=\rho^{(i-j)^{2}}$ that correspond to suburban/rural environments with small angular spreads at the transmitter [11]. Unless

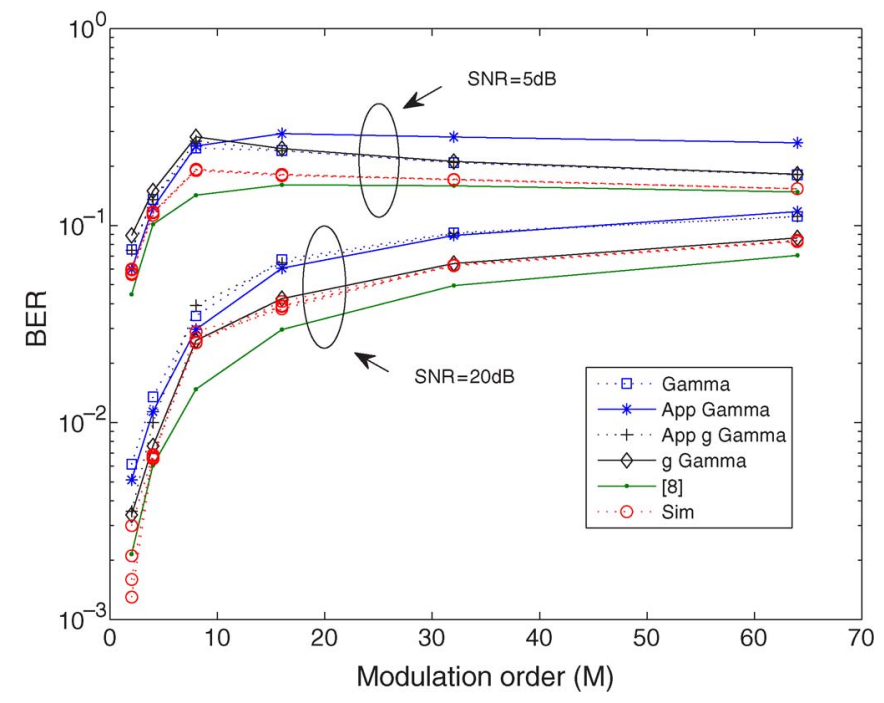

Fig. 1. Average BER for a $4 \times 4$ MIMO with precoding, several modulations (represented by modulation order $M$ ), and SNR $=5$ and 20 dB. $\rho=0$.

otherwise stated, the power matrix $\mathbf{P}$ is obtained with $\pi_{i}=1, i=$ $1, \ldots, n_{\mathrm{T}}$, and the precoding matrix $\mathbf{V}$ contains the eigenvectors of $E\left\{\mathbf{H}_{\mathbf{c}}^{\dagger} \mathbf{H}_{\mathbf{c}}\right\}=\mathbf{R}_{\mathbf{t}}$ (3). For a rather general class of channel models [12], this matrix is, in fact, the capacity-achieving precoder $\mathbf{V}$ when the receiver has perfect instantaneous channel-state information [channel-state information at the receiver (CSIR)], but the transmitter has only access to its distribution [channel distribution information at the transmitter (CDIT)]. This precoder is also shown to be optimal from an SINR standpoint with a linear MMSE receiver [2].

Fig. 1 shows the average BER of each transmitted stream with different modulation schemes when $\rho=0$ and SNR is $5 \mathrm{~dB}$ or $20 \mathrm{~dB}$. The four theoretical expressions here developed are represented as follows:

1) Gamma approximation (Gamma);

2) High-SNR Gamma approximation (App Gamma);

3) Generalized Gamma approximation (g Gamma);

4) Low-SNR generalized Gamma approximation (App g Gamma).

We can see that, for high SNR, the generalized Gamma approximation fits extremely well the simulation results (the curves labeled with Sim represent the four transmitted streams in the $4 \times 4$ system), whereas the other three approximations slightly overestimate the average BER. For low SNR, the fit is not very accurate, but still, the generalized Gamma gives the best approximation, together with its low-SNR approximation (App g Gamma). In addition, the values obtained in [8] are shown in this figure, where we have included the effect of the precoder. We can see that they are not accurate for high SNR.

Fig. 2 shows the average BER of each transmitted stream with quadrature phase-shift keying (QPSK) modulation when $\rho=0.9$ and SNR is varied between $0 \mathrm{~dB}$ and $30 \mathrm{~dB}$. For this value of $\rho$, the BER of the four transmitted streams get very dissimilar values due to the different SINRs induced by the precoder, suggesting the importance of the ability to properly choose the transmitted powers. Because they are the most accurate and for clarity, here, we only represent the generalized Gamma and its low-SNR approximation. We can see that, again, the generalized Gamma produces very close values to the simulation results for all SNR values. On the other hand, the average BER obtained in [4] under the normal approximation assumed large antenna dimensions, and we can see that it is clearly not accurate for a small number of antennas. 


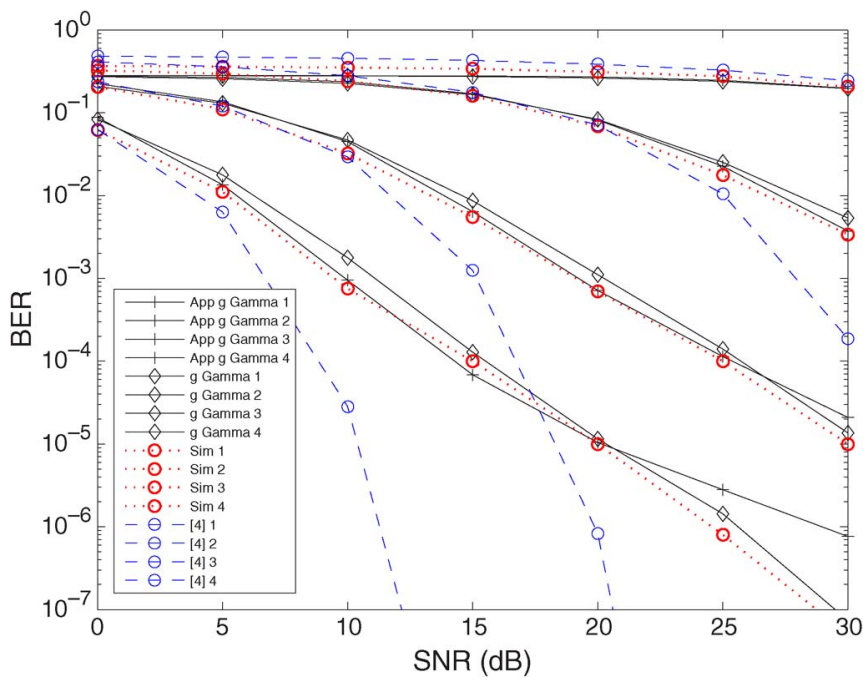

Fig. 2. Average BER for a $4 \times 4$ MIMO with precoding, QPSK modulation, and several values of SNR. $\rho=0.9$ : analytical versus simulated values.

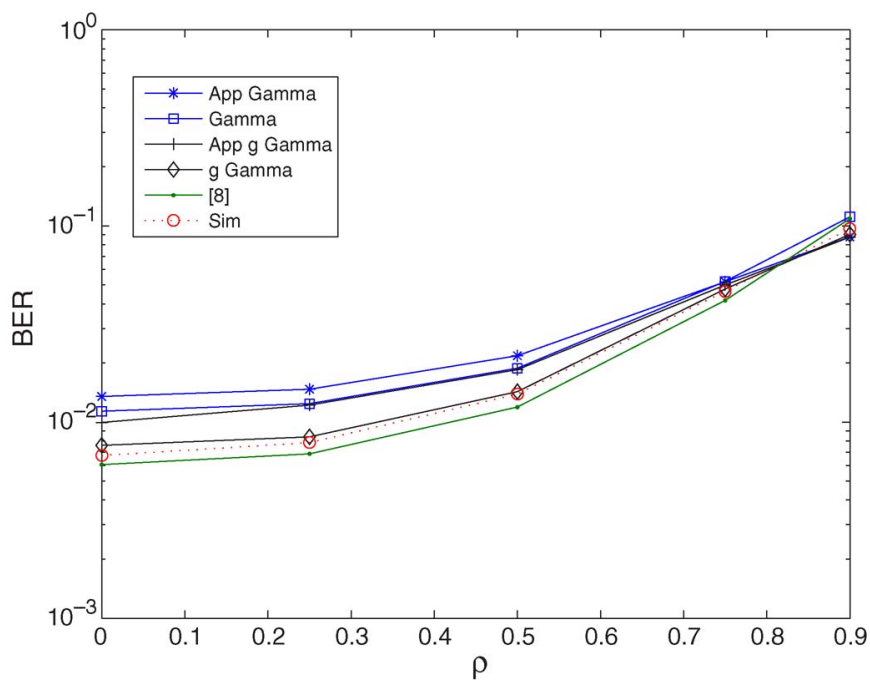

Fig. 3. Average BER (also averaged over the four transmitted streams) for a $4 \times 4$ MIMO with precoding, QPSK modulation, and SNR $=20 \mathrm{~dB}$.

Fig. 3 shows the average BER, averaged over the four transmitted streams, with QPSK modulation when $\mathrm{SNR}=20 \mathrm{~dB}$ and different values of $\rho$. We can see that the generalized Gamma gives a very good fit for all values of $\rho$. In [8], correlation is assumed only at the receiver side; therefore, we should expect it to be accurate only for the smallest values of $\rho$. However, even for these values, we can see that our expressions give a better fit to the simulation results, because [8] is not accurate for high-SNR values.

The results shown considered a uniform power allocation. In Fig. 4, the average BER is shown for a nonuniform power allocation example, where the values of the $\pi_{i}$ are set to $1.8,1.2,0.6$ and 0.4 ; therefore, the stream that is transmitted through the worst channel is enhanced with a higher power (i.e., the effect of the channel is somehow compensated by the power allocation). We can again see a good fit of the analytical and simulation results.

\section{CONCLUSION}

We have obtained analytical expressions for the average BER of MIMO systems in transmit-correlated Rayleigh flat-fading channels

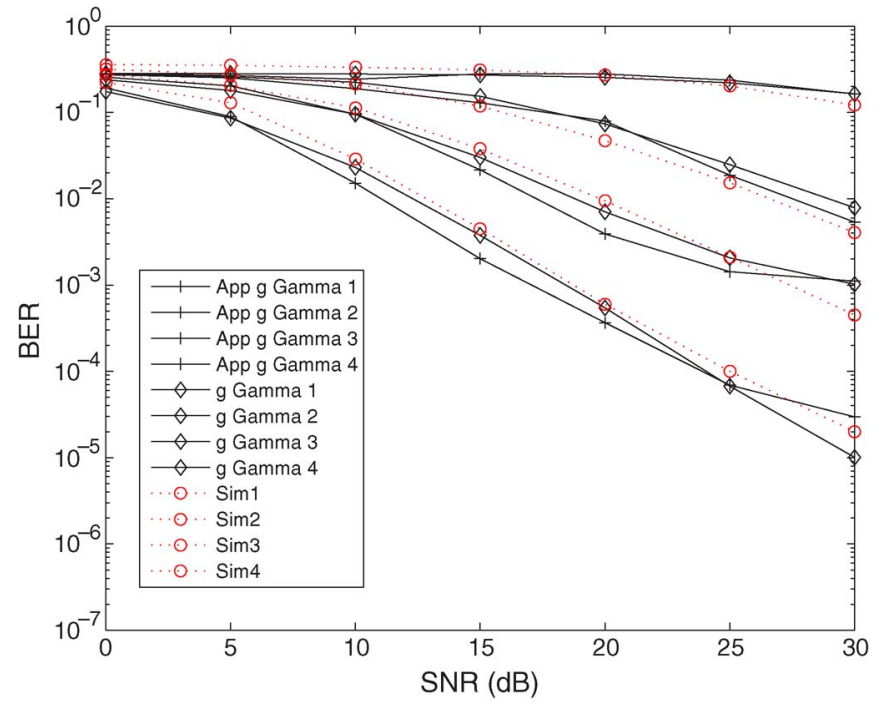

Fig. 4. Average BER for a $4 \times 4$ MIMO with precoding and nonuniform power [ $\left.\begin{array}{llll}1.8 & 1.2 & 0.6 & 0.4\end{array}\right]$, with QPSK modulation and several values of SNR. $\rho=0.9$ : analytical versus simulated values.

with or without precoding and with MMSE receivers. These expressions, whose accuracy even for small dimensions has been shown by comparison with numerical results, can be used to optimize the transmitter for a given target BER or, in general, as a useful tool for the system design.

\section{APPENDIX A}

\section{Average Bit Error Rate Using a Generalized GAMMA DISTRIBUTION}

The MGF of a random variable $S$ with probability distribution function $f_{S}(s)$ is

$$
M_{S}(x)=\int_{-\infty}^{\infty} e^{s x} f_{S}(s) d s
$$

In the particular case of the SINR following a generalized Gamma distribution $f_{g g}(s)$, we have

$$
\frac{1}{2 \sqrt{\pi}} M_{g g}(x)=\frac{1}{2 \sqrt{\pi}} \int_{0}^{\infty} e^{s x} f_{g g}(s) d s .
$$

For 2-PAM, we can see that the average BER of (13) is [1]

$$
\bar{P}_{e} \approx \frac{1}{2 \sqrt{\pi}} \int_{0}^{\infty} e^{-s} f_{g g}(s) d s=\frac{1}{2 \sqrt{\pi}} M_{g g}(-1) .
$$

Based on [7, eqs. (69) and (70)], $\bar{P}_{e}$ can be obtained, if $\xi>1$, as

$$
\frac{1}{2 \sqrt{\pi}} M_{T}(-1)=\frac{1}{2 \sqrt{\pi}} \exp \left(\frac{\alpha\left(1-(1+\theta \xi)^{\frac{\xi-1}{\xi}}\right)}{\xi-1}\right) \text {. }
$$

In addition, if $\xi<1$, we have

$$
\frac{1}{2 \sqrt{\pi}} M_{T}(-1)=\frac{1}{2 \sqrt{\pi}} \exp \left(\frac{\alpha\left(\left(\frac{1}{1+\theta \xi}\right)^{\frac{1-\xi}{\xi}}-1\right)}{1-\xi}\right) \text {. }
$$


The BER of 4-QAM and $M$-QAM can be also obtained following the same procedure.

\section{APPENDIX B \\ AVErage Bit ERror RATE FOR LOW SIGNAL-TO-NOISE RATIO}

At low SNR, the Gamma distribution and the generalized Gamma distribution are very close. Thus, $\xi \approx 1$. Based on [13, eq. (2.6)], we know that the expectation of $e^{-w t^{\xi}}$ under the generalized Gamma distribution is

$$
E\left(e^{-w s^{\xi}}\right)=\frac{\left(\frac{1}{\theta}\right)^{\alpha \xi}}{\left(\left(\frac{1}{\theta}\right)^{\xi}+w\right)^{\alpha}}
$$

which, for $\xi \approx 1$, becomes

$$
E\left(e^{-w s}\right) \approx\left(\left(\frac{1}{\theta}\right)^{\xi}+w\right)^{-\alpha}\left(\frac{1}{\theta}\right)^{\alpha \xi}
$$

For 2-PAM, the average BER is obtained, setting $w=1$, as

$$
\begin{aligned}
\bar{P}_{e} & =\frac{1}{2 \sqrt{\pi}} \int_{0}^{\infty} e^{-s} f_{g g}(s) d s=\frac{1}{2 \sqrt{\pi}} E\left(e^{-s}\right) \\
& =\frac{1}{2 \sqrt{\pi}}\left(\left(\frac{1}{\theta}\right)^{\xi}+1\right)^{-\alpha}\left(\frac{1}{\theta}\right)^{\alpha \xi} .
\end{aligned}
$$

The BER of 4-QAM and $M$-QAM can be also obtained following the same procedure.

\section{REFERENCES}

[1] A. García Armada, L. Hong, and A. Lozano, "Bit loading for MIMO with statistical channel information at the transmitter and ZF receivers," in Proc. IEEE Int. Conf. Commun., Dresden, Germany, Jun. 2009, pp. 1-5.

[2] A. Scaglione, G. Giannakis, and S. Barbarossa, "Redundant filterbank precoders and equalizers I: Unification and optimal designs," IEEE Trans. Signal Process., vol. 47, no. 7, pp. 1988-2006, Jul. 1999.

[3] D. Gore, R. W. Heath, Jr., and A. Paulraj, "On performance of the zeroforcing receiver in presence of transmit correlation," in Proc. IEEE Int. Symp. Inf. Theory, Lausanne, Switzerland, Jul. 2002, p. 159.

[4] D. Guo, S. Verdú, and L. K. Rasmussen, "Asymptotic normality of linear multiuser receiver outputs," IEEE Trans. Inf. Theory, vol. 48, no. 12, pp. 3080-3095, Dec. 2002.

[5] M. Kiessling and J. Speidel, "Analytical performance of MIMO MMSE receivers in correlated Rayleigh fading environments," in Proc. IEEE Veh. Technol. Conf., Oct. 2003, vol. 3, pp. 1738-1742.

[6] A. L. Moustakas, K. R. Kumar, and G. Caire, "Performance of MMSE MIMO receivers: A large $\mathrm{N}$ analysis for correlated channels," in Proc. Veh. Technol. Conf., Dec. 2009, vol. 1, pp. 1-5.

[7] P. Li, D. Paul, R. Narasimhan, and J. Cioffi, "On the distribution of SINR for the MMSE MIMO receiver and performance analysis," IEEE Trans. Inf. Theory, vol. 52, no. 1, pp. 271-286, Jan. 2006.

[8] A. Kammoun, M. Kharouf, W. Hachem, and J. Najim, "BER and outage probablity approximations for LMMSE detectors on correlated MIMO channels," IEEE Trans. Inf. Theory, vol. 55, no. 10, pp. 4386-4397, Oct. 2009.

[9] W. Hachem, O. Khorunzhiy, P. Loubaton, J. Najim, and L. Pastur, "A new approach for capacity analysis of large dimensional multiple-antenna channels," IEEE Trans. Inf. Theory, vol. 54, no. 9, pp. 3987-4004, Sep. 2008

[10] A. Paulraj, Introduction to Space-Time Wireless Communications. Cambridge, U.K.: Cambridge Univ. Press, 2003.

[11] A. M. Tulino, A. Lozano, and S. Verdú, "Impact of antenna correlation on the capacity of multiantenna channels," IEEE Trans. Inf. Theory, vol. 51, no. 7, pp. 2491-2509, Jul. 2005.
[12] A. M. Tulino, A. Lozano, and S. Verdú, "Capacity-achieving input covariance for single-user multiantenna channels," IEEE Trans. Wireless Commun., vol. 5, no. 3, pp. 662-671, Mar. 2006.

[13] P. H. Huang and T. Y. Hwang, "On new moment estimation of parameters of the generalized gamma distribution using its characterization," Taiwanese J. Math., vol. 10, no. 4, pp. 1083-1093, Jun. 2006. 